\title{
Phase Evolution of the Electronic Transmission Through a Kondo Correlated Quantum Dot
}

\author{
R. Franco, J. Silva-Valencia, \\ *Departamento de Física, Universidad Nacional de Colombia, A.A. 5997, Bogotá, Colombia \\ and M. S. Figueira \\ * Instituto de Física, Universidade Federal Fluminense, \\ Avenida Litorârea s/n, 24210-346, Niterói, Rio de Janeiro, Brazil
}

Received on 8 December, 2005

\begin{abstract}
We study the scattering phase shift of the Kondo assisted transmission through a quantum dot (QD), considering a model that includes an additional non resonant channel transmission. To compute the phase evolution and the transmission amplitude of the QD, for different temperatures, we describe the QD employing the single Anderson impurity model in the limit of infinity Coulomb repulsion, within the X-boson approach. Our results are consistent with the development of an unusually large phase evolution at around $\pi$ in the Kondo valley, observed in recent experiments, and is consistent with others theoretical treatments.
\end{abstract}

Keywords: Quantum dots; Scattering phase; Kondo effect; X-boson

\section{INTRODUCTION}

The scattering phase shift of the Kondo effect cannot be accessed in bulk Kondo systems or in mesoscopic systems by means of conductance measurements [1-3]. It was theoretically predicted that the Kondo scattering in the unitary limit (the localized occupation number $n_{f} \approx 1$ ), of the impurity Anderson model induces a phase shift of $\pi / 2$ [4]. However, recent experimental results for a quantum dot (QD) embedded in a double path electronic interferometer [1,2], presents anomalous behavior, as plateaus of the phase shift at around $\pi$. Recent theoretical work [5], employing the Anderson impurity model to describe the QD and using the numerical renormalization group (NRG) technique, predicts the existence of a $\pi / 2$ plateaus for the phase evolution of the QD in the Kondo limit. This prediction disagrees with the experimental reports of Y. Ji et.al. [2] which measures the complex transmission amplitude of a QD near the unitary limit and observed that the transmission phase evolves almost linearly over a range of about $1.5 \pi$, when the Fermi energy was scanned through a spin degenerate energy level of the QD. Another theoretical treatment, developed to describe the same problem [6], employs the Kondo model for a single and multilevel QD and using scale arguments obtain results that agree well with the experiments $[1,2]$ in the Coulomb blockade regime, but fail to explain the large span of the phase shift in the Kondo regime [2]. Other argument suggested that the stochastic fluctuation of the Kondo temperature $\left(T_{K}\right)$ due to topology, finite size effects and mesoscopic fluctuations, should originate serious limitations to determine the transmission phase through a QD [7].

We consider a model that includes a weak direct nonresonant transmission through a QD, represented in Fig. 1 by the path $W$ as well as a Kondo-resonant transmission channel represented in the same figure by the leads connected to the quantum dot by the hopping term $V$. This model was previously discussed employing the slave boson mean field technique (SBMFT) [8]. The importance of including more than one resonant transmission channel is that the Fano-type

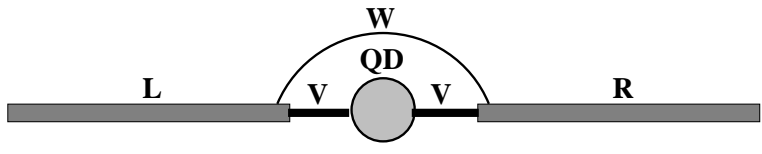

FIG. 1: Pictorial view of the embedded QD in the leads. The hopping between the leads and the QD is represented by the letter $V$ and the tunneling amplitude $W$ is responsible for the direct transmission between the left and right leads.

interference between the resonant and the non-resonant transmission channel can provide a possible explanation for the anomalous phase drop accompanied by transmission zero [911]. Our model is formally equivalent to a two terminal $A B$ interferometer containing a QD [12], but in this work the phase shift is not computed and the reference arm is associated with our direct transmission term $W$. It is necessary to highlight, that in our treatment the non-resonant direct channel, describes the direct transmission through the QD. The phase shift computed, takes into account the transmission through all the system (resonant transmission of the QD channel, and the $W$-direct channel) and not only through the QD [5].

We applied the X-boson method, for the single impurity case, to describe the transport properties of this system. As we analyzed in our previous works $[13,14]$, the impurity X-boson approach is not a reliable approximation, at very low temperatures, in the extreme Kondo regime but it is a good approximation, at low temperatures, in the intermediate valence and empty dot regime. At temperatures $T \approx T_{K}$ or higher, the impurity X-boson approach is a good alternative to the SBMFT in all the regimes, because the SBMFT presents a spurious second order phase transition at $T_{K}$ [17], whereas the X-boson solution evolves to the correct thermodynamic limit at high temperatures [13]. 


\section{MODEL}

We employ the model Hamiltonian $H=H_{L}+H_{R}+H_{D}+H_{T}$ to describe the QD system, represented in a pictorial form in Fig. 1. The Hamiltonian for the left (L) and right (R) leads are given by

$$
H_{\alpha}=\sum_{\mathbf{k}, \alpha} E_{\mathbf{k}, \alpha} c_{\mathbf{k}, \alpha, \sigma}^{\dagger} c_{\mathbf{k}, \alpha, \sigma} \quad(\alpha=L, R),
$$

where $c_{\mathbf{k}, \alpha, \sigma}^{\dagger}\left(c_{\mathbf{k}, \alpha, \sigma}\right)$ is a creation (destruction) operator of an electron with energy $E_{\mathbf{k}, \alpha}$, momentum $\mathbf{k}$ and spin $\sigma$ on the lead $\alpha$. The interacting QD is described by

$$
H_{D}=\sum_{\sigma} E_{d, \sigma} X_{d, \sigma \sigma}
$$

where this term describes the Anderson impurity characterized by a localized $d$ level $E_{d, \sigma}$, (we employ the $d$ letter to indicate the localized electrons at the impurity site). We work in the infinite correlation limit $U=\infty$, because this limit is able to catch all the relevant physics of the Kondo effect and the Yang Ji et.al. experiment $[1,2]$ were performed in a strong correlated QD $(U=1.5 \mathrm{meV})$ [15]. We shall employ the Hubbard operators [16] to project out the doubly occupied state $|d, 2\rangle$ from the local states of the impurity. The identity decomposition in the reduced space of impurity local states is given by $X_{d, 00}+X_{d, \sigma \sigma}+X_{d, \overline{\sigma \sigma}}=I$, where $\bar{\sigma}=-\sigma$, and the three $X_{d, a a}$ are the projectors into the corresponding states $|d, a\rangle$. The occupation numbers on the impurity $n_{d, a}=<X_{d, a a}>$ satisfy the "completeness" relation $n_{d, 0}+n_{d, \sigma}+n_{d, \bar{\sigma}}=1$. The tunneling Hamiltonian $H_{T}$ is

$$
\begin{aligned}
H_{T}= & \sum_{\alpha=L, R} \sum_{\mathbf{k}, \sigma}\left(V_{\alpha} X_{d, 0 \sigma}^{\dagger} c_{\mathbf{k}, \alpha, \sigma}+H . c .\right) \\
& +\sum_{\mathbf{k}, \mathbf{k}^{\prime}, \sigma}\left(W c_{L, \mathbf{k}, \sigma}^{\dagger} c_{R, \mathbf{k}^{\prime}, \sigma}+H . c .\right) .
\end{aligned}
$$

The tunneling amplitude $W$ is responsible for the direct transmission between the two leads, and $V_{\alpha}$ for the tunneling between the QD and the lead $\alpha$.

For simplicity, we assume symmetric junctions (i.e. $V_{L}=$ $\left.V_{R}=V\right)$ and identical leads. The direct tunneling matrix element $W=|W| \exp (i \eta)$, while the hopping matrix elements $V_{\alpha}$ can be kept as positive real numbers without loss of generality. The $\eta$ stands for the phase difference between the resonant and the non resonant component and here we assume that $\eta=0, \pi[8]$.

Using the Friedel's relation between the scattering matrix and the local Green's functions (GF) $[4,8]$ one can write the transmission amplitude of the electrons with energy $E$ from the left to the right lead as

$$
\begin{aligned}
t_{L R}(E)= & \operatorname{iexp}(i \eta)\left|t_{b}\right|+i \exp (i \eta) \Gamma_{e f f} \tilde{G}_{Q D}(E) \\
& {\left[\left|r_{b}\right| \cos (\eta)-i\left(\left|t_{b}\right|+\sin (\eta)\right],\right.}
\end{aligned}
$$

where $\left|t_{b}\right|=2 x /\left(1+x^{2}\right)$ with $x=\pi \rho_{c} W$ is the amplitude of the direct transmission, with $\rho_{c}$ being the conduction density of states of the leads and $\left|r_{b}\right|$ is obtained by the relation $\left|t_{b}\right|^{2}+\left|r_{b}\right|^{2}=1$. $\tilde{G}_{Q D}(E)=V^{2} D_{\sigma} G_{f}(E) G_{c}^{2}(E)$ is the local dressed GF, that describes a QD embedded between the two leads, with $V$ being the hybridization, $D_{\sigma}$ is a variational X-boson parameter, $G_{f}(E)$ and $G_{c}(E)$ are the GF's of the QD and the conduction band, represented here by the leads. These GF's are obtained by the X-boson approach $[13,14]$. The effective hybridization parameter is obtained by $\Gamma_{e f f}=\Gamma /\left(1+x^{2}\right)$, with $\Gamma=2 \pi \rho_{c} V^{2}$. In our approximation we employ the local GF for the QD, obtained using the $\mathrm{X}$-boson treatment for the impurity case $[13,14]$.

The limit of unitary direct transmission $\left(\left|t_{b}\right| \sim 1\right)$, was previously investigated by us for a ballistic quantum wire coupled to a QD [13]. At finite temperatures, we consider the thermal average of the transmission amplitude

$$
t_{L R}=\int\left(-\frac{\partial n_{f}}{\partial E}\right) t_{L R}(E) d E
$$

where $n_{f}$ is the Fermi-Dirac distribution function.

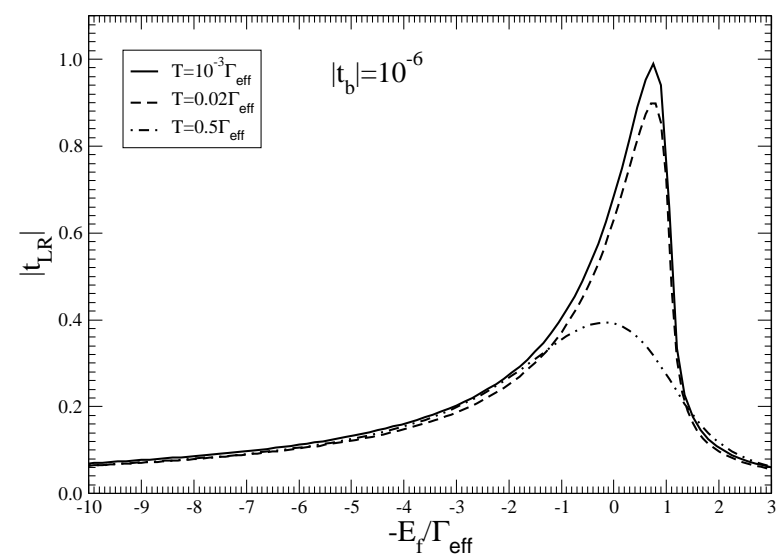

FIG. 2: Magnitude of the transmission amplitude $\left|t_{L R}\right|$ for $\eta=0$ and $\left|t_{b}\right|=10^{-6}$ vs. $-E_{f} / \Gamma_{e f f}$, for different temperatures.

\section{RESULTS AND DISCUSSION}

To simplify the calculations we represent the leads by a square conduction band of half-width $D$ and we employ in the numerical calculations the following parameters set: $D=100 \Delta$ with $\Delta=\pi V^{2} / 2 D=1,\left|t_{b}\right|=10^{-6}$ (figures 1-2), $\left|t_{b}\right|=0.2$ (figures 3-6) and $\eta=0$ in all the figures.

In Fig. 2 we show the magnitude of the transmission amplitude $\left|t_{L R}\right|$, for a very low value of the direct transmission probability $\left|t_{b}\right|^{2}=10^{-12}$ vs. $-E_{f} / \Gamma_{\text {eff }}$ (the level energy in the QD) for different temperature values. The results are consistent with the situation where we have an embedded QD, because in this case the direct transmission is $W=0$. The maximum value of the $\left|t_{L R}\right|$ occurs in the Kondo limit and when 


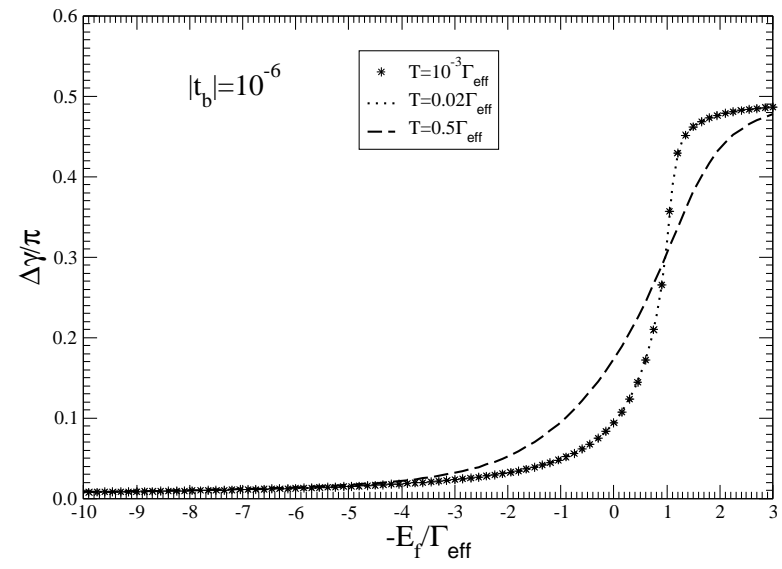

FIG. 3: The phase shift $\Delta \gamma$ for $\eta=0$ and $\left|t_{b}\right|=10^{-6}$ vs. $-E_{f} / \Gamma_{e f f}$, for different temperatures.

the temperature increases, the Kondo effect vanishes and the maximum decreases.

In Fig. 3 we present the phase shift $\Delta \gamma / \pi$ vs. $-E_{f} / \Gamma_{e f f}$, for different temperatures and the same parameter set of Fig. 2. The results are consistent with the expected $\pi / 2$ phase change in the Kondo limit.

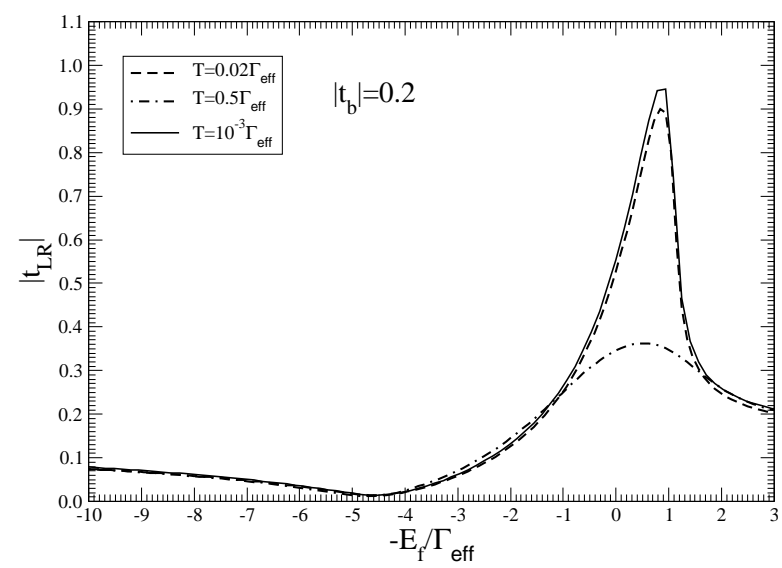

FIG. 4: The magnitude of the transmission amplitude $\left|t_{L R}\right|$ vs. $-E_{f} / \Gamma_{e f f}$, for different temperatures.

In Fig. 4 we present the magnitude of the transmission amplitude $\left|t_{L R}\right|$, for the same parameter set of Fig. 2, but now employing $\left|t_{b}\right|=0.2$ (finite transmission probability for the direct channel). Comparing the figures 2 and 4 we can see that the transmission $\left|t_{L R}\right|$ presents little variation in both figures what agrees with the results presented in reference [8] but in Fig. 5 we present the corresponding phase shift $\Delta \gamma / \pi$ vs. $-E_{f} / \Gamma_{\text {eff }}$ and we obtain a unusually large plateau of the phase evolution at around $\pi$. As we work in the strong Coulomb correlation limit $U \rightarrow \infty$ when the QD total occupation number is closest to its maximum value $n_{Q D}=1$, we describe the phase shift in the Coulomb blockade valley regime. Our results show that in this situation the phase shift $\Delta \gamma / \pi$ follows the Coulomb blockade regime presented in a recent experimental report $[1,2]$,

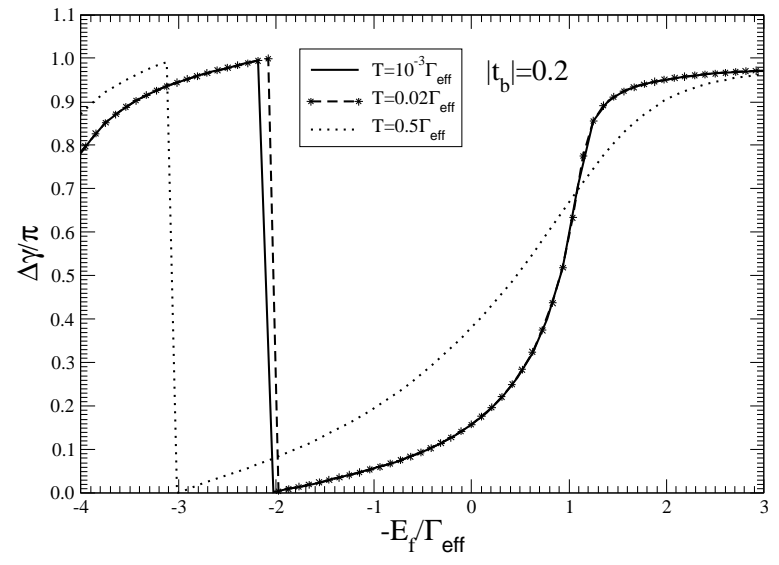

FIG. 5: The phase shift $\Delta \gamma$ vs. $-E_{f} / \Gamma_{e f f}$, for different temperatures.

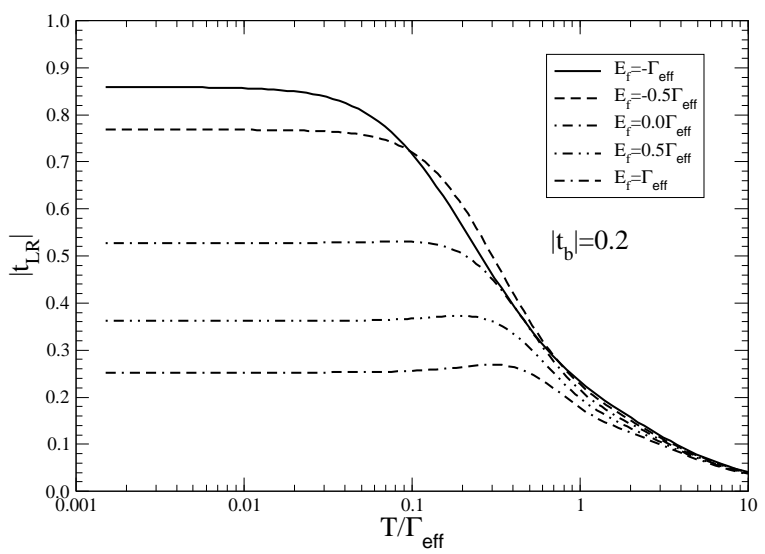

FIG. 6: The magnitude of the transmission amplitude $\left|t_{L R}\right|$ vs. $T / \Gamma_{e f f}$, for different regimes of the QD.

and in a theoretical work [8].

In Fig. 6 we show the magnitude of the transmission amplitude $\left|t_{L R}\right|$ vs. temperature $T / \Gamma_{e f f}$, for different values of $E_{f}$, associated with different regimes of the QD; empty dot, intermediate valence and Kondo. Our results agree qualitatively with those expected for an embedded QD [5, 18]. In Fig. 7 we present the phase shift $\Delta \gamma$ vs. the $E_{f}$ level energy in the QD. For $E_{f}<0 ; E_{f}=-0.5 \Gamma_{e f f}$ and $E_{f}=-\Gamma_{e f f}$, (Kondo regime) the $\Delta \gamma / \pi$ magnitude is closer to 0.5 , at low temperatures, which agrees with the results for the phase shift of the conventional impurity Kondo problem. At temperatures greater than the $T_{K}$, for all the cases the phase shift is closest to the value 0.8 , which agrees with our result for the Coulomb blockade regime.

\section{CONCLUSIONS}

The quantum interference process between a weak probability of direct transmission $\left|t_{b}\right|$ and the Kondo resonant channel, is responsible for the changes into the transmission $\left|t_{L R}\right|$ and the phase shift scattering $\Delta \gamma$, this later in a more important 


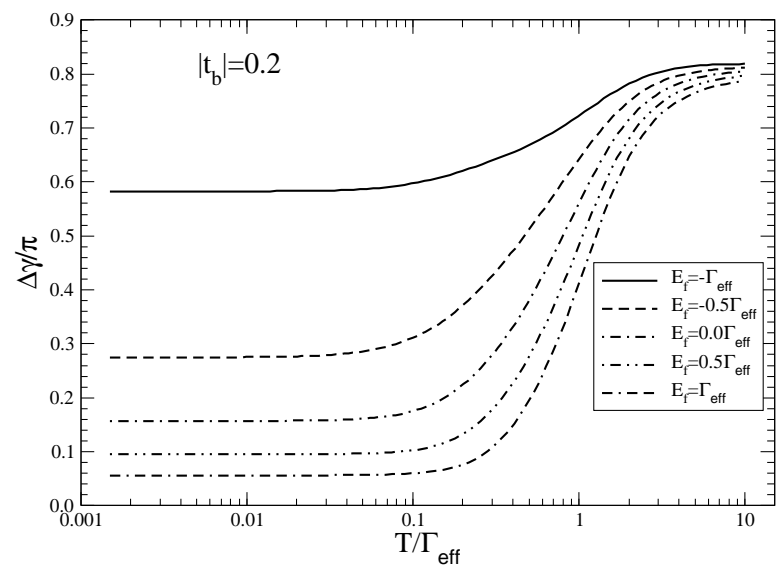

FIG. 7: The phase shift of the transmission amplitude $\Delta \gamma \mathrm{vs.} T / \Gamma_{e f f}$, for different regimes of the QD. way, producing plateaus of $\Delta \gamma$ near $\pi$, as function of the QD energy $E_{f}$. These results agree with the experimental report of Y. Ji et.al. [1,2] in the transition from the Kondo to Coulomb blockade regime.

\section{Acknowledgments}

We acknowledge the financial support of the Colombia National University, DINAIN-project:20601003550 and DIBproject:803954, CNPq(Brazilian National Research Council) and FAPERJ (Rio de Janeiro State Research Foundation), from the grant "Primeiros projetos".
[1] Y. Ji, M. Heiblum, D. Sprinzak, D. Mahalu, and H. Shtrikman, Science 290, 779 (2000).

[2] Y. Ji, M. Heiblum, and D. Shtrikman, Phys. Rev. Lett. 88, 076601 (2002).

[3] W. G. Van der Wiel, S. De Franceschi, T. Fujisawa, J. M. Elzerman, S. Tarucha, and L. P. Kouwenhoven, Science 289, 2105 (2000).

[4] D. C. Langreth, Phys. Rev. 150, 516 (1966).

[5] U. Gerland, J. von Delft, T. A. Costi, and Y. Oreg. Phys. Rev. Lett. 84, 3710 (2000).

[6] P. G. Silvestrov and Y. Imry. Phys. Rev. Lett. 90, 106602 (2003).

[7] C. H. Lewenkopf and H. A. Weidenmüller. Phys. Rev. B 71, 121309(R) (2005).

[8] K. Kang, Mahn-Soo Choi, and Seongjae Lee, Phys. Rev. B 71, 045330 (2005).
[9] H. Xu and W. Sheng Phys. Rev. B 57, 11903 (1998)

[10] P. S. Deo, Solid State Comm. 107, 69 (1998).

[11] C. M. Ryu and S. Y. Cho, Phys. Rev. B 58, 3572 (1998).

[12] B. R. Bulka and P. Stefański, Phys. Rev. Lett. 86, 5128 (2001).

[13] R. Franco, M. S. Figueira, and E. V. Anda, Phys. Rev. B 67, 155301 (2003).

[14] R Franco, M. S. Figueira, and M. E. Foglio, Phys. Rev. B 66, 045112 (2002).

[15] Yigal Meir, Ned S. Wingreen, and Patrick A. Lee, Phys. Rev. Lett. 70, 2601 (1993). 84, 3710 (2000).

[16] J. Hubbard, Proc. R. Soc. London, Ser. A 77, 237 (1964).

[17] P. Coleman, Phys. Rev. B 29,3035 (1984); Phys. Rev. B. 35, 5072 (1987).

[18] T. A. Costi, Phys. Rev. B 64, 241310 (2001). 